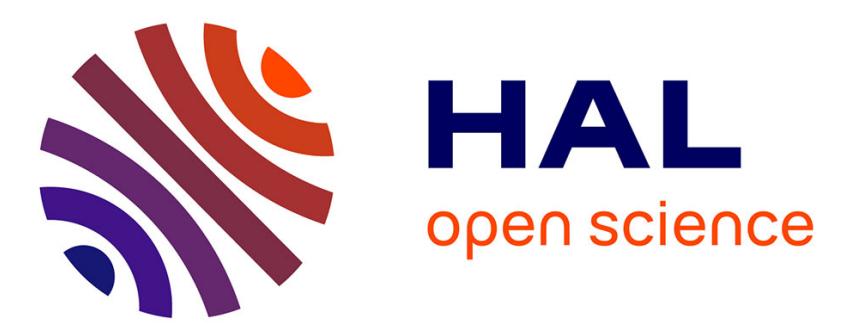

\title{
Étude exploratoire des caractéristiques professionnelles d'un échantillon de suicidants hospitalisés
}

Stéphane François, Jean-Bernard Garré, Marie-Pierre Guiho-Bailly, Aurélie François, Chantal Bertin, Julie Bodin, Bénédicte Gohier, Yves Roquelaure

\section{- To cite this version:}

Stéphane François, Jean-Bernard Garré, Marie-Pierre Guiho-Bailly, Aurélie François, Chantal Bertin, et al.. Étude exploratoire des caractéristiques professionnelles d'un échantillon de suicidants hospitalisés. Santé Publique, 2011, 23 (2), pp.101-112. hal-03389584

\section{HAL Id: hal-03389584 \\ https://univ-angers.hal.science/hal-03389584}

Submitted on 21 Oct 2021

HAL is a multi-disciplinary open access archive for the deposit and dissemination of scientific research documents, whether they are published or not. The documents may come from teaching and research institutions in France or abroad, or from public or private research centers.
L'archive ouverte pluridisciplinaire HAL, est destinée au dépôt et à la diffusion de documents scientifiques de niveau recherche, publiés ou non, émanant des établissements d'enseignement et de recherche français ou étrangers, des laboratoires publics ou privés. 


\title{
ÉTUDE EXPLORATOIRE DES CARACTÉRISTIQUES PROFESSIONNELLES D'UN ÉCHANTILLON DE SUICIDANTS HOSPITALISÉS
}

\author{
Stéphane François et al.
}

S.F.S.P. | Santé Publique

2011/2 - Vol. 23

pages 101 à 112

ISSN 0995-3914

Article disponible en ligne à l'adresse:

http://www.cairn.info/revue-sante-publique-2011-2-page-101.htm

Pour citer cet article :

François Stéphane et al., «Étude exploratoire des caractéristiques professionnelles d'un échantillon de suicidants hospitalisés »,

Santé Publique, 2011/2 Vol. 23, p. 101-112.

Distribution électronique Cairn.info pour S.F.S.P..

(C) S.F.S.P.. Tous droits réservés pour tous pays.

La reproduction ou représentation de cet article, notamment par photocopie, n'est autorisée que dans les limites des conditions générales d'utilisation du site ou, le cas échéant, des conditions générales de la licence souscrite par votre établissement. Toute autre reproduction ou représentation, en tout ou partie, sous quelque forme et de quelque manière que ce soit, est interdite sauf accord préalable et écrit de l'éditeur, en dehors des cas prévus par la législation en vigueur en France. II est précisé que son stockage dans une base de données est également interdit. 


\title{
Étude exploratoire des caractéristiques professionnelles d'un échantillon de suicidants hospitalisés
}

\author{
Exploratory study about professional characteristics \\ of suicide attempters
}

\author{
Stéphane François (1), Jean-Bernard Garre (2), Marie-Pierre Guiho-Bailly(1), \\ Aurélie François ${ }^{(3)}$, Chantal Bertin ${ }^{(1)}$, Julie Bodin (1), Bénédicte Gohier ${ }^{(3)}$, \\ Yves Roquelaure ${ }^{(1)}$
}

\begin{abstract}
Résumé : Cette étude a pour objectif de décrire les caractéristiques professionnelles d'un échantillon de suicidants. Un enquêteur a interrogé les suicidants âgés de 18 à 65 ans, hospitalisés consécutivement dans une unité spécialisée du CHU d'Angers sur une durée de 6 mois et demi. Au total, 87 suicidants actifs avec un emploi ont été interrogés. Ils ont souvent été confrontés à des contraintes organisationnelles décrites dans la littérature comme responsables de souffrance mentale liée au travail. Cela concerne globalement autant les hommes que les femmes. En comparaison aux enquêtes de santé au travail (Sumer, Samotrace...), les suicidants sont plus nombreux à ressentir entre autres un stress intense au travail, une conscience professionnelle heurtée et à être en situation tendue selon le modèle de Karasek. Cela pourrait être en faveur d'un lien entre les tentatives de suicide et certains facteurs de pénibilité mentale au travail. Les résultats de cette étude sont à interpréter avec prudence du fait des phénomènes de circularité des données et de la faiblesse de l'échantillon.
\end{abstract}

Mots-clés : Tentatives de suicide - suicide - conditions travail - organisation travail - travail facteur psycho social.

Summary: We aimed to describe the professional characteristics of suicide attempters. One investigator asked 87 consecutive patients, aged 18 to 25 years, who had been hospitalized in a university hospital in Angers after having attempted suicide anytime during a 6.5-month period (November 2008-April 2009) to complete a questionnaire about their working conditions. The working conditions of both sexes were approximatively the same. Many patients described situations where work organization was known to produce given mental disorders. Patients hospitalized for suicide attempts are in professions that leaves them more stressed than the general population of workers (Sumer, Samotrace etc.). This evidence could favour a link between attempted suicide and mental factors of strain at work. The results of this study should be interpreted with caution because of the phenomena of circuitous data and small sample size.

Keywords: Suicide attempt - suicide - depression - organization - work - social support.

(1) Laboratoire d'ergonomie et d'épidémiologie en santé au travail - Faculté de médecine d'Angers - 1, rue haute de reculée, 49045 Angers cedex.

(2) Département de psychiatrie et de psychologie médicale 4, rue Larrey, CHU d’Angers, 49933 Angers Cedex 9.

(3) Consultation de pathologie professionnelle 4, rue Larrey, CHU d'Angers, 49933 Angers Cedex 9. 
La prévention du suicide est une priorité de santé publique en France. En effet, le suicide est la cause de 10600 décès par an sur la période 20012003 [1]; mais, selon l'Inserm, ce nombre serait sous estimé de 20 à $25 \%[2,3]$. Parmi les facteurs de risque de suicide, la souffrance au travail semble représenter une part non négligeable [4-6]. Le contexte de mondialisation et d'intensification croissante des conditions de travail a entraîné une évolution profonde du travail à travers son organisation. Les collectifs de travail permettant la mise en place de stratégies défensives laissent souvent place à l'isolement du salarié. Dans cette course au progrès permanent, la réalisation du travail selon les valeurs techniques et déontologiques de métier n'est pas toujours possible. Ce contexte est d'autant plus délétère pour la santé si la dynamique de la reconnaissance est altérée [7].

Lorsque ces situations se prolongent, elles peuvent être à l'origine de véritables décompensations psycho-pathologiques. Le suicide en est l'exemple le plus violent. Il intervient lorsque les stratégies de défense et d'adaptation sont dépassées, lorsque les ressources internes et environnementales sont insuffisantes et que le salarié ne voit plus de solution à ses difficultés. Le suicide, comme les tentatives de suicide (TS) et les idées suicidaires définissent le phénomène suicidaire. Bien que très médiatisé depuis "les affaires du techno centre de Renault Guyancourt » et malgré quelques études [8-11], la crise suicidaire liée au travail reste peu documentée. Le gouvernement a affirmé sa volonté de mieux définir ce phénomène, en termes quantitatif et qualitatif [12]. L'objectif de cette étude est d'analyser les caractéristiques professionnelles d'une population de suicidants.

\section{Matériel et méthode}

\section{Population étudiée}

L'enquête a été réalisée par questionnaires auprès de suicidants, hospitalisés consécutivement à l'unité médico-psychosociale (UMPS) du CHU d'Angers qui prend en charge des suicidants après leur hospitalisation en réanimation ou aux urgences médicales adultes. Les patients mineurs et/ou présentant une psychose n'y sont pas admis et sont pris en charge dans d'autres unités spécialisées. Cette étude s'est déroulée sur la période du 2 novembre 2008 au 15 mai 2009. Les suicidants exclus de l'étude étaient ceux ayant fugué ou quitté le service de soin contre avis médical et n'ayant pas rendu le questionnaire, ceux présentant des troubles de conscience et ceux ayant déjà été inclus dans l'étude lors d'une TS antérieure. Le sujet de cet article concerne uniquement les réponses aux questionnaires portant sur les caractéristiques professionnelles des suicidants actifs avec un emploi.

\section{Recueil des données}

Un auto-questionnaire proposé par un enquêteur avait pour objectif de réaliser une description précise des conditions de travail. Il était basé sur des questionnaires existants, utilisés en santé au travail (Karasek, Samotrace et Siegrist) et un questionnaire intitulé Travail et santé mentale créé par les investigateurs. 
Le questionnaire de Karasek est composé de 26 questions. Il explore ainsi trois dimensions : la demande psychologique (le fait de devoir apprendre des choses nouvelles, d'avoir un travail qui nécessite un haut niveau de compétence, d'avoir une quantité excessive de travail à réaliser), la latitude décisionnelle (par exemple avoir la possibilité d'influencer le déroulement du travail, de pouvoir prendre des décisions, de pouvoir faire preuve de créativité), et enfin le soutien social au travail (rapports coopératifs ou collaboratifs avec les collègues et la hiérarchie). La demande psychologique et la latitude décisionnelle permettent de situer le salarié dans une des quatre catégories suivantes: détendu (faible demande psychologique et forte latitude décisionnelle), actif (forte demande psychologique et forte latitude décisionnelle), passif (faible demande psychologique et faible latitude décisionnelle) ou tendu (forte demande psychologique et faible latitude décisionnelle). Cette dernière catégorie est appelée job strain. Le fait de travailler dans cette situation expose davantage au risque de développer des pathologies cardio-vasculaires, des troubles musculo-squelettiques et des troubles psychiatriques comme la dépression [13]. La situation de job strain dans un contexte de faible soutien professionnel correspond à la situation de travail la plus péjorative dite iso strain. Les scores ont été dichotomisés à partir des médianes nationales issues de l'enquête Sumer $2003[14,15]$.

Le questionnaire Samotrace est issu d'un système national de surveillance épidémiologique de la santé mentale en lien avec le travail [16]. Son volet épidémiologique en entreprise utilisé ici, permet de préciser la nature de l'emploi et certaines conditions de travail (catégorie socioprofessionnelle, type de contrat de travail, horaires, expositions à des contraintes physiques, à des discriminations, ou encore le fait d'avoir sa conscience professionnelle heurtée...).

Le questionnaire de Siegrist recherche la présence d'un déséquilibre dans le rapport investissement-récompense au travail, qui favorise l'apparition de pathologies $[17,18]$. Pour une question de faisabilité, nous n'avons utilisé que sept items de ce questionnaire. Il s'agissait, entre autres, du fait d'estimer avoir des perspectives de promotions suffisantes, un salaire suffisant, le respect de la part de sa hiérarchie ou de ses collègues, d'être traité de façon injuste au travail...

Le questionnaire Travail et santé mentale recherchait la présence « d'organisations pathogènes du travail " décrites dans la littérature $[8,13,16,19]$ que le patient aurait rencontrées dans les 12 derniers mois. Les éléments de l'organisation examinés étaient, entre autres, la surcharge de travail, les délais à respecter, les ordres contradictoires, le manque de moyens, les prescriptions floues...

L'avis du suicidant sur l'existence d'un lien entre le travail et le geste suicidaire a été recherché mais sera analysé au cours de futurs travaux.

Dans le but d'obtenir des réponses valables aux questionnaires, ces derniers étaient posés 48 heures après la TS afin de limiter la présence d'effets toxiques neurologiques, notamment cognitifs, en cas d'intoxication médicamenteuse volontaire. 


\section{Analyses des données}

Les analyses statistiques ont été réalisées avec le logiciel SPSS version 17. La distribution de l'échantillon en fonction du genre et de l'âge, a été comparée à celle des Pays de la Loire et de la France, à l'aide du test du Chi 2 d'ajustement pour le genre et d'un test de comparaison de moyennes pour l'âge. Le seuil de signification a été fixé à 0,05 et tous les tests étaient bilatéraux.

Ensuite, une analyse descriptive a été réalisée sur les conditions de travail des 87 suicidants actifs avec un emploi de l'étude, selon le genre et l'âge. Du fait de la faiblesse de l'échantillon, les analyses en fonction de l'âge sont proposées selon deux classes, de 18 à 39 ans et de 40 à 59 ans. Pendant l'étude, il n'y a pas eu de suicidants actifs avec un emploi, âgés de plus de 59 ans. En ce qui concerne la description des conditions de travail en fonction de l'âge, les résultats significativement différents entre les classes d'âge ont

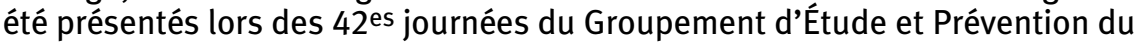
Suicide [20].

\section{Résultats}

Au total, 150 suicidants ont été interrogés. Parmi eux, seulement six ont refusé de participer à l'étude, ne se sentant pas en état de répondre ou estimant que le questionnaire ne les concernait pas.

87 suicidants actifs avec un emploi (57 femmes et 30 hommes) ont répondu aux questionnaires relatifs au travail. La moyenne d'âge de l'échantillon est de $35 \pm 15$ ans. Environ les deux tiers (65\%) sont âgés de 18 à 39 ans et $35 \%$ de 40 à 59 ans. Les réponses aux questions présentaient peu de données manquantes (de 0 à 9,2\% selon les questions).

\section{Analyse du questionnaire}

Les principales catégories socio-professionnelles représentées sont les employés ( $29 \%)$, les ouvriers ( $28 \%$ ) et les professions intermédiaires $(25 \%)$. Les hommes sont en majorité ouvriers, tandis que les femmes appartiennent davantage aux catégories employés et professions intermédiaires. Les CDI sont les plus fréquents et un cinquième de l'échantillon est en CDD (cf. tableau I).

Le temps travaillé par semaine dépasse souvent 35 heures $(\geq 59 \%$ des cas), mais on observe davantage de travail à temps partiel parmi les femmes. Entre un quart et un tiers des hommes et des femmes travaillent plus de 10 heures par jour et plus d'un samedi sur deux.

Les suicidants sont nombreux à se dire exposés à des contraintes physiques et/ou psychologiques au travail telles que les exigences du public, les postures contraignantes, le port de charges, les expositions aux poussières... (cf. tableau II).

Parmi les éléments d'organisations pathogènes du travail, les rotations, la surcharge de travail, les délais à respecter, les dérangements fréquents et les injonctions contradictoires sont les plus souvent évoqués mais ne sont pas les seuls (cf. tableau II). 
Tableau I : Caractéristiques sociodémographiques et professionnelles

\begin{tabular}{|l|r|r|r|r|r|r|}
\hline \multirow{2}{*}{ Variables } & \multicolumn{2}{c|}{ Hommes $(n=30)$} & \multicolumn{2}{c|}{ Femmes $(n=57)$} & \multicolumn{2}{c|}{ Total $(n=87)$} \\
\cline { 2 - 7 } & $n$ & $\%$ & $n$ & $\%$ & $n$ & $\%$ \\
\hline Classe d'âge & & & & & & \\
$18-39$ ans & 17 & 63 & 35 & 66 & 52 & 65 \\
$40-59$ ans & 10 & 37 & 18 & 34 & 28 & 35 \\
Diplôme & & & & & & \\
Aucun & 7 & 24 & 6 & 10 & 13 & 15 \\
BEPC & 2 & 7 & 6 & 11 & 8 & 9 \\
CAP OU BEP & 14 & 48 & 17 & 30 & 31 & 36 \\
Bac & 5 & 17 & 11 & 19 & 16 & 19 \\
Bac+2 & 1 & 4 & 5 & 9 & 6 & 7 \\
Plus que Bac +2 & 0 & 0 & 12 & 21 & 12 & 14 \\
CSP & & & & & & \\
Employés & 5 & 17 & 19 & 35 & 24 & 29 \\
Ouvriers & 14 & 48 & 9 & 17 & 23 & 28 \\
Professions intermédiaires & 2 & 7 & 19 & 35 & 21 & 25 \\
Cadres, P.I.S * & 4 & 14 & 5 & 9 & 9 & 11 \\
Artisans, commerçants et & 2 & 7 & 2 & 4 & 4 & 5 \\
chefs d'entreprise & & & & & \\
Agriculteur exploitant & 23 & 80 & 37 & 70 & 60 & 73 \\
Contrat de travail & 3 & 10 & 13 & 25 & 16 & 20 \\
CDI & 3 & 10 & 3 & 5 & 6 & 7 \\
CDD & 2 & 7 & 0 & 0 & 2 & 2 \\
Autres & & & & & & \\
\hline
\end{tabular}

* Professions intellectuelles supérieures.

Les suicidants sont nombreux à ressentir un stress intense au travail (55\% des hommes et $54 \%$ des femmes). Les rapports tendus avec la hiérarchie concernent un grand nombre (35\%) de suicidants (cf. tableau II). Les tensions avec les collègues sont aussi évoquées. Les rapports sociaux dans l'entreprise peuvent être assez dégradés. Ainsi, $24 \%$ des hommes et $30 \%$ des femmes se disent menacés ou humiliés au travail.

Le travail est réalisé d'une façon contraire à l'éthique professionnelle pour $31 \%$ des hommes et $24 \%$ des femmes.

Les rétributions matérielles et symboliques en reconnaissance du travail accompli semblent souvent insuffisantes. Le salaire est jugé insatisfaisant pour $62 \%$ des hommes et $46 \%$ des femmes. Le poste occupé n'est pas à la hauteur du niveau de qualification pour plus d'un quart de l'échantillon. De même, les perspectives de promotion ou d'évolution de carrière semblent insatisfaisantes dans un tiers des cas environ (cf. tableau II).

Au total, huit questionnaires de Karasek sur les 87 proposés présentaient des données manquantes et n'étaient donc pas exploitables. On observe une prédominance des situations tendues au travail parmi la catégorie des artisans, commerçants, chefs d'entreprises, celle des professions intermédiaires, celle des ouvriers et celle des employés. Le faible nombre d'agriculteurs $(n=2)$ ne permet pas d'analyse. Les femmes sont davantage en situation tendue au travail (cf. tableau III). 
Tableau II : Conditions de travail et vécu du travail selon le genre

\begin{tabular}{|c|c|c|c|c|c|c|}
\hline \multirow{2}{*}{ Variables } & \multicolumn{2}{|c|}{ Hommes $(n=30)$} & \multicolumn{2}{|c|}{ Femmes $(n=57)$} & \multicolumn{2}{|c|}{ Total $(n=87)$} \\
\hline & $n$ & $\%$ & $n$ & $\%$ & $n$ & $\%$ \\
\hline \multicolumn{7}{|l|}{ Temps de travail } \\
\hline Temps partiel & 4 & 13 & 19 & 33 & 23 & 28 \\
\hline Travailler plus de $10 \mathrm{~h}$ par jour & 7 & 24 & 15 & 26 & 22 & 26 \\
\hline Lever avant $5 \mathrm{~h}$ pour le travail & 6 & 21 & 8 & 14 & 14 & 16 \\
\hline Travailler plus d'1 dimanche/2 & 3 & 10 & 11 & 19 & 14 & 16 \\
\hline Trajet domicile-travail $>1 \mathrm{~h}$ aller et retour & 6 & 21 & 7 & 13 & 13 & 16 \\
\hline Coucher après minuit pour le travail & 3 & 10 & 9 & 16 & 12 & 14 \\
\hline Travailler la nuit & 1 & 3 & 5 & 9 & 6 & 7 \\
\hline \multicolumn{7}{|l|}{ Contraintes physiques et psychologiques } \\
\hline Postures pénibles & 15 & 52 & 26 & 46 & 41 & 48 \\
\hline Ports de charges lourdes & 14 & 48 & 24 & 42 & 38 & 44 \\
\hline Exposition aux poussières ou aux fumées & 13 & 45 & 11 & 19 & 24 & 28 \\
\hline Travail répétitif & 8 & 28 & 12 & 21 & 20 & 24 \\
\hline Exposition aux produits chimiques & 10 & 35 & 9 & 16 & 19 & 22 \\
\hline Bruits intenses & 8 & 28 & 10 & 18 & 18 & 21 \\
\hline Travail posté & 5 & 17 & 7 & 12 & 12 & 14 \\
\hline Exposition aux intempéries & 7 & 24 & 4 & 7 & 11 & 13 \\
\hline \multicolumn{7}{|l|}{ Facteurs d'organisation pathogènes du travail } \\
\hline Supporter les exigences du public & 14 & 48 & 35 & 65 & 49 & 59 \\
\hline Rotations & 13 & 43 & 30 & 53 & 43 & 51 \\
\hline Surcharge de travail & 12 & 41 & 28 & 52 & 40 & 48 \\
\hline Délai à respecter & 17 & 59 & 21 & 39 & 38 & 46 \\
\hline Souvent dérangé & 13 & 45 & 24 & 44 & 37 & 45 \\
\hline Travail en sous-effectif & 10 & 35 & 22 & 41 & 32 & 39 \\
\hline Injonctions contradictoires & 12 & 41 & 20 & 37 & 32 & 39 \\
\hline Manque de moyens & 8 & 28 & 20 & 37 & 28 & 34 \\
\hline Rentabilité difficile à atteindre & 8 & 28 & 20 & 37 & 28 & 34 \\
\hline Qualité difficile à atteindre & 12 & 41 & 14 & 26 & 26 & 32 \\
\hline Tâches mal définies & 9 & 31 & 16 & 30 & 25 & 30 \\
\hline Système d'évaluation non professionnelle & 5 & 17 & 19 & 37 & 24 & 30 \\
\hline Glissements de tâches & 5 & 17 & 8 & 15 & 13 & 16 \\
\hline Mutation subie & 3 & 10 & 8 & 15 & 11 & 13 \\
\hline Sous-charge de travail & 1 & 3 & 4 & 7 & 5 & 6 \\
\hline \multicolumn{7}{|l|}{ Vécu, ambiance et relations au travail } \\
\hline Stress intense & 16 & 55 & 29 & 54 & 45 & 54 \\
\hline Tension avec la hiérarchie & 8 & 28 & 21 & 39 & 29 & 35 \\
\hline Menaces ou humiliations & 7 & 24 & 16 & 30 & 23 & 28 \\
\hline Conscience professionnelle heurtée & 9 & 31 & 13 & 24 & 22 & 27 \\
\hline Tension avec les collègues & 6 & 21 & 13 & 24 & 19 & 23 \\
\hline Avertissement & 4 & 14 & 9 & 17 & 13 & 16 \\
\hline Menace de licenciement & 3 & 10 & 8 & 15 & 11 & 13 \\
\hline Discriminations liées à l'âge & 1 & 3 & 7 & 13 & 8 & 10 \\
\hline Violences physiques & 1 & 3 & 4 & 7 & 5 & 6 \\
\hline Discriminations liées au handicap & 0 & 0 & 4 & 7 & 4 & 5 \\
\hline \multicolumn{7}{|l|}{ Reconnaissance au travail } \\
\hline Salaires insatisfaisants & 18 & 62 & 25 & 46 & 43 & 52 \\
\hline Promotions insatisfaisantes & 9 & 31 & 20 & 39 & 29 & 36 \\
\hline $\begin{array}{l}\text { Je ne reçois pas le respect que je mérite } \\
\text { de mes supérieurs }\end{array}$ & 8 & 28 & 20 & 37 & 28 & 34 \\
\hline Formation initiale non concordant au poste & 8 & 28 & 17 & 32 & 25 & 30 \\
\hline Traitement injuste au travail & 8 & 28 & 12 & 22 & 20 & 24 \\
\hline $\begin{array}{l}\text { Je ne reçois pas le respect que je mérite } \\
\text { de mes collègues }\end{array}$ & 5 & 17 & 10 & 19 & 15 & 18 \\
\hline
\end{tabular}


Tableau III : Répartition des situations de travail décrites par le modèle de Karasek, selon la CSP, le sexe et l'âge

\begin{tabular}{|c|c|c|c|c|}
\hline & $\begin{array}{c}\text { Actifs } \\
n\end{array}$ & $\begin{array}{c}\text { Passifs } \\
n\end{array}$ & $\begin{array}{c}\text { Détendus } \\
n\end{array}$ & $\begin{array}{c}\text { Tendus } \\
n\end{array}$ \\
\hline \multicolumn{5}{|l|}{ CSP } \\
\hline Agriculteurs exploitants & 0 & 2 & 0 & 0 \\
\hline Artisans, commerçants, chefs d'entreprises & 2 & 0 & 0 & 2 \\
\hline Cadres, professions intellectuelles supérieures & 5 & 1 & 1 & 2 \\
\hline Professions intermédiaires & 2 & 5 & 1 & 11 \\
\hline Employés & 4 & 4 & 2 & 13 \\
\hline Ouvriers & 4 & 3 & 3 & 12 \\
\hline \multicolumn{5}{|l|}{ Sexe } \\
\hline Homme & 9 & 6 & 3 & 9 \\
\hline Femme & 8 & 9 & 4 & 31 \\
\hline \multicolumn{5}{|l|}{ Âge } \\
\hline $18-39$ & 9 & 9 & 4 & 28 \\
\hline $40-59$ & 8 & 6 & 3 & 12 \\
\hline Ensemble & 17 & 15 & 7 & 40 \\
\hline
\end{tabular}

\section{Discussion}

\section{Comparaison des caractéristiques " genre et âge " de l'échantillon} à celles des populations de la France et des Pays de la Loire

Les descriptions dans la littérature concernant les populations de suicidants correspondent à des populations « jeunes et féminines »[21, 22]. On constate dans notre échantillon une moyenne d'âge plus jeune et une proportion de femmes supérieure à celles des populations de référence (cf. tableau IV).

Tableau IV : Comparaison de l'échantillon aux populations de référence selon les sources de l'INSEE

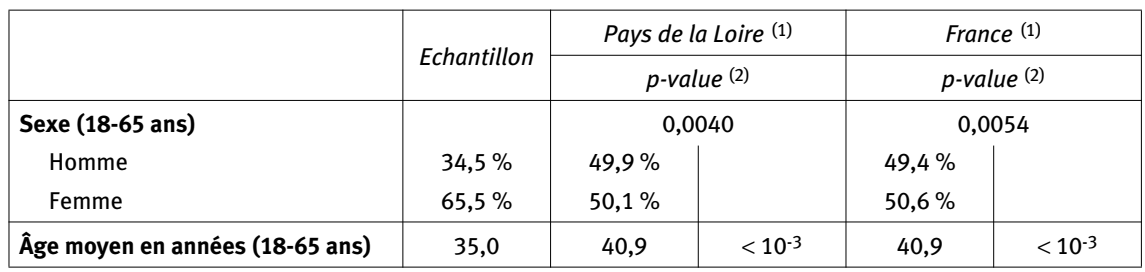

(1) Données correspondantes aux années 2006-2007 selon les chiffres les plus récents proposés par l'INSEE au moment de l'étude [31].

(2) Comparaison de la population concernée à celle de l'échantillon de l'étude.

\section{Intérêts et limites de l'étude}

L'étude a été réalisée à l'unité médico-psycho-sociale du CHU d’Angers qui est le principal service d'accueil en urgence des crises suicidaires dans le Maine-et-Loire, ce qui permet un bon recrutement des suicidants dans ce 
département, bien que certains ne soient pas admis dans ce service (admission dans un autre service et TS non hospitalisées). Cette étude a permis une description détaillée des caractéristiques professionnelles d'une population de suicidants. De plus, l'utilisation des mêmes questionnaires de santé au travail par notre enquête et d'autres études (Sumer, Samotrace...), permet de comparer les caractéristiques professionnelles de travailleurs hospitalisés pour TS à celles de populations au travail.

Une limite majeure de cette étude est relative au phénomène de circularité des données, à savoir l'influence de l'état dépressif sur l'appréciation des conditions de travail et de l'environnement en général [23]. En effet, une partie non négligeable de l'échantillon était atteinte d'un syndrome dépressif [20] et ceci a pu entraîner une vision plus péjorative des conditions de travail. Enfin, le nombre d'inclus est faible. L'ensemble de ces éléments implique de réaliser une interprétation prudente des résultats.

\section{Conditions de travail selon l'âge}

Le vécu des contraintes de travail par les travailleurs vieillissants a été bien décrit par l'enquête ESTEV. Il s'agit de l'impression d'accroissement de la difficulté avec l'âge de toutes les exigences rencontrées dans le travail [24]. Cela est secondaire à de multiples modifications physiologiques accompagnant le vieillissement. Notre étude a mis en évidence que les suicidants âgés entre 40 et 59 ans sont plus nombreux à considérer être exposés à une surcharge de travail, une qualité difficile à atteindre, une rentabilité difficile à atteindre et des délais à respecter [20]. Ces résultats amènent à s'interroger sur la question de l'adaptation du travail à l'homme, et de la prise en compte des caractéristiques intrinsèques des salariés par l'organisation du travail. On observe cependant que les suicidants âgés de 40 à 59 ans ne sont pas davantage exposés à la situation tendue décrite par le modèle de Karasek (cf. tableau III). On sait par ailleurs que de manière générale, les travailleurs vieillissants ont développé des ficelles de métier, du fait de leur expérience, leur permettant de mieux organiser leur travail et de mieux faire face aux aléas. Cependant, les nouvelles organisations du travail évoluent de plus en plus vite et disqualifient les savoir-faire acquis. Elles offrent de moins en moins la possibilité aux travailleurs vieillissants d'acquérir de l'expérience et de pallier aux évolutions physiologiques liées à l'âge.

\section{Contrats de travail, CSP et tentatives de suicide}

C. Cohidon et al. ont mis en évidence une prépondérance de TS parmi les intérimaires [21]. Dans notre échantillon, nous retrouvons une proportion de CDD $(19,5 \%)$ supérieure à celles des populations de la France $(8,4 \%)$ et des Pays de la Loire ( $9 \%)$. Cela pourrait être en faveur d'un lien possible entre le statut professionnel précaire et les TS.

Les ouvriers et employés sont les plus touchés par les TS, suivis des professions intermédiaires. Ces résultats sont proches de ceux décrits dans la littérature épidémiologique [4,21]. Les hommes de l'échantillon sont davantage des ouvriers, tandis que les femmes sont en majorité des employées et professions intermédiaires. La catégorie agriculteur est la 
moins représentée dans notre échantillon (seulement deux TS) ce qui pouvait être attendu puisqu'il est décrit dans la littérature que les agriculteurs réalisent peu de TS mais présentent le plus fort taux de suicide. L'accès à des méthodes plus violentes et les difficultés socio-économiques expliquent en partie ces données [21] qui peuvent être nuancées par un potentiel biais de déclaration. Les artisans, commerçants et chefs d'entreprises sont également peu représentés. Il est difficile d'établir un lien entre les CSP et les phénomènes suicidaires. En effet, toutes les catégories semblent concernées, certaines davantage par les suicides et d'autres par les tentatives de suicide. Il est possible en revanche que l'instabilité de l'emploi en cas de contrat de travail de type CDD ou interim, ainsi que de mauvaises conditions de travail (cf. paragraphe ci-dessous) pèsent plus que la catégorie socioprofessionnelle dans les processus suicidaires.

\section{Les conditions de travail mentionnées par les suicidants}

Un stress intense est ressenti au travail par la moitié de l'échantillon, ce qui représente un pourcentage deux fois plus grand que ceux donnés par la littérature concernant les populations au travail $[25,26]$.

La surcharge de travail souvent évoquée par les suicidants est reconnue par de nombreuses études comme l'une des causes de stress les plus importantes [25].

Le niveau de rentabilité est souvent difficile à atteindre selon eux, d'autant plus s'il y a des délais à respecter. Le travail est favorable à la santé lorsque le compromis entre la préservation de celle-ci et les exigences de la tâche, est possible. Une intensité du travail trop forte ne le permet pas [19].

L'ambiguiité de rôle, quand la tâche est mal définie et que les injonctions sont contradictoires, est également un facteur de stress [25].

«Le dérangement fréquent dans le travail correspond à une pénibilité mentale relevant des dissonances cognitives, par exemple lorsqu'il s'agit d'abandonner une tâche pour une autre plus urgente »[27]. Ces conditions de travail sont souvent accompagnées d'un manque de moyens humains.

Avoir la conscience professionnelle heurtée concerne $27 \%$ de l'échantillon, ce qui est nettement plus élevé que dans l'échantillon Samotrace qui en retrouvait $12 \%[16]$. Cet élément est associé à une détresse psychique détectée par le General Health Questionnaire avec un odd ratio égal à 1,57 chez les hommes [28]. Il s'agit ici d'une souffrance éthique qui concerne des travailleurs poussés à réaliser des tâches contraires à la conception du travail bien fait, développée par le groupe professionnel auquel ils adhèrent [27]. Pouvoir réaliser un travail que l'on juge de qualité selon ses valeurs est fondamental dans la construction de la santé psychique.

Des menaces ou humiliations au travail sont évoquées par un nombre plus important de suicidants ( $24 \%$ des hommes et $30 \%$ des femmes) que de salariés interrogés dans l'enquête Samotrace (10\% des hommes et 13,5\% des femmes) [16].

Les conflits avec les collègues et la hiérarchie sont délétères. Dans de telles ambiances de travail, l'absence de confiance entre collègues ne permet pas la mise en place de liens de coopération. Les salariés se retrouvent isolés. 
Selon P. Davezies, les suicides liés au travail sont « l'aboutissement extrême de ce mouvement d'individualisation » [29].

Les horaires atypiques peuvent perturber la vie familiale, les activités culturelles et la vie sociale [19]. Ils ont un impact direct sur ces dernières et peuvent fragiliser le soutien provenant de la sphère privée. Les horaires de travail s'accordent bien (ou très bien) avec les engagements familiaux et sociaux pour $67 \%$ de l'échantillon, pourcentage plus faible que ceux rencontrés dans la littérature concernant les populations au travail [16].

Enfin, des systèmes de promotion, d'avancement de carrière basés sur des modèles d'évaluation analysant incomplètement le travail sont décrits par de nombreux suicidants. L'évaluation complète du travail implique la mise en visibilité de la mobilisation subjective du travailleur. Parmi les différentes méthodes, l'évaluation individualisée des performances s'avère certainement la plus délétère. Cette dernière contient une part d'arbitraire pouvant engendrer un sentiment d'injustice et d'isolement [30].

\section{Une fréquence élevée de situations tendues au travail selon le modèle de Karasek}

Comme dans l'enquête Sumer 2003, la situation de job strain (ou tendue) concerne davantage les femmes qui sont en plus grande proportion que les hommes à être exposées à une faible latitude décisionnelle, ainsi que les catégories ouvriers et employés quelque soit le sexe [14]. Cependant, on observe que notre échantillon est deux fois plus touché par cette situation que la population au travail interrogée par l'enquête Sumer 2003 [14]. De plus, la situation de tension au travail n'est pas la seule à être associée au stress professionnel. Les effets stressants de la catégorie active au travail définie par le modèle de Karasek ont été montrés dans la littérature [14]. La catégorie active concerne $21 \%$ des suicidants. Ces résultats montrent l'importance tenue par l'équilibre entre demande psychologique, latitude décisionnelle et soutien professionnel dans la genèse des processus suicidaires.

\section{Reconnaissance de l'investissement au travail : un élément crucial}

"La reconnaissance est la forme spécifique de la rétribution moralesubjective accordée à ego en contrepartie de sa contribution à l'efficacité de l'organisation du travail, c'est-à-dire à l'engagement de sa subjectivité et de son intelligence » [31]. Elle peut être d'ordre matériel (salaires, promotions...) ou symbolique (respect des supérieurs, des collègues, reconnaissance de l'effort fourni...). On note un pourcentage important de suicidants à estimer que la reconnaissance professionnelle est insuffisante à la vue de tous leurs efforts puisqu'ils sont nombreux à estimer que leur salaire est insuffisant, que leur poste n'est pas à la hauteur de leurs qualifications et qu'ils sont traités injustement au travail. La reconnaissance du travail réel, par les pairs et la hiérarchie est en effet très importante puisqu'elle permet la construction identitaire [32]. 


\section{Conclusion}

L'intérêt majeur de cette étude est d'avoir permis une description précise des caractéristiques professionnelles d'une population de suicidants. On note que ces derniers sont nombreux à se dire exposés à des éléments d'organisations pathogènes du travail et des rapports sociaux dégradés dans l'entreprise. Les femmes sont davantage en situation de job strain. Parmi les conditions de travail connues pour être responsables de souffrance psychique $[13,33]$, certaines sont retrouvées à une fréquence plus élevée que dans les enquêtes concernant les populations au travail [14, 26, 28]. Il s'agit entre autres du stress intense ressenti au travail, de la conscience professionnelle heurtée, de la situation tendue selon le modèle de Karasek et des menaces ou humiliations subies au travail. Cela pourrait être en faveur d'un lien entre les TS et les facteurs de pénibilité mentale au travail.

Une interprétation prudente des résultats s’impose à la vue du faible effectif de l'échantillon, et par la présence des syndromes dépressifs pouvant majorer les réponses négatives, notamment concernant le travail.

Il serait intéressant afin de donner du poids à ces résultats de savoir si l'échantillon étudié est bien représentatif des populations habituelles de suicidants et éventuellement de disposer d'une population témoin pour comparer leurs conditions de travail.

Enfin, il convient de rappeler que les suicides et TS liées au travail ne sont que la partie visible de l'iceberg, puisque bien plus nombreux sont les passages à l'acte évités de justesse par l'intervention du médecin du travail, du médecin traitant ou parce que le travailleur s'est présenté de lui-même dans un service de soins [34].

\section{BIBLIOGRAPHIE}

1. Base nationale des causes médicales de décès 2001-2003. Inserm CépiDc, exploitation Fnors-ORS.

2. Chappert JL, Pequignot F, Jougla E. Évaluation de la qualité des données de mortalité par suicide : biais et impacts sur les données nationales en France, à partir des causes indéterminées quant à l'intention. Série Études, Drees (30), 2003.

3. Jougla $E$, Péquignot $F$, Le Toullec A, Bovet $M$, Mellah $M$. « Données et caractéristiques épidémiologiques de la mortalité par suicide ", Actualité et dossier en santé publique, 2003;45:31-4.

4. Gournay M, Lanièce F, Kryvenac I. Étude des suicides liés au travail en Basse-Normandie. Travailler 2004(12):91-8.

5. Moreira P, Prolongeau H. Travailler à en mourir : Flammarion ; 2009. 243.

6. Peze M. $2^{\mathrm{e}}$ ed. Ils ne mouraient pas tous mais tous étaient frappés. Paris : Flammarion ; 2010. 214.

7. Dejours C, Bègue F. Suicide et travail : que faire ? 1' éd. Paris : PUF ; 2009.129.

8. Amagasa T, Nakayama T, Takahashi Y. Karojisatsu in Japan: characteristics of 22 cases of work-related suicide, J. Occup. Health 2005;47:157-64.

9. Hawton K, Harris L, Simkin S, Bale E, Bond A. Social class and suicidal behaviour: the associations between social class and the characteristics of deliberate self-harm patients and the treatment they are offered. Soc Psychiatry Psychiatr Epidemiol 2001;36:437-43.

10. Koskinen O, Pukkila K, Hakko H, Tiihonen J, Väisänen E, Särkioja T et al. Is occupation relevant in suicide? J Affect Disord 2002;70:197-203.

11. Nishimura M, Terao T, Soeda S, Nakamura J, Iwata N, Sakamoto K. Suicide and occupation: further supportive evidence for their relevance, Prog Neuropsychopharmacol Biol Psychiatry 2004;28:83-7.

12. Nasse $P$, Legeron $P$. Rapport sur la détermination, la mesure et le suivi des risques psychosociaux au travail. France. Ministère du travail, des relations sociales et de la solidarité. 2008. 42. Disponible à l'adresse URL:http://www.travailler-mieux.gouv.fr/IMG/pdf/RAPPORT_FINAL_.pdf 
13. Bonde JPE. Psychosocial factors at work and risk of depression :asystematic review of the epidemiological evidence. Occup. Environ. Med. 2008;65:438-45.

14. Guignon N, Niedhammer I, Sandret N. Les facteurs psychosociaux au travail. Une évaluation par le questionnaire de Karasek dans l'enquête Sumer 2003. DARES 2008(22,1).

15. Karasek RA. Job demands, job decision latitude and mental strain: implications for job redesign. Admin Science Quart.1979;24:285-307.

16. Cohidon C, Murcia M et al. Samotrace - Volet «Épidémiologie en entreprise ». Résutats intermédiaires à un an (3 000 questionnaires). Régions Centre, Pays de la Loire et Poitou-Charentes et le Comité de pilotage de Samotrace. Collection «Santé et travail ». Mai 2007.

17. Niedhammer J. Siegrist. Facteurs psychosociaux au travail et maladies cardio-vasculaires : l'apport du modèle Déséquilibre Efforts/Récompenses. Rev. Epidem. Et Santé Publ 1998;46:398-410.

18. Siegrist J, Starke D, Chandola T, Godin I, Marmot M, Niedhammer I, Peter R. The measurement of effortreward imbalance at work: European comparisons. Soc Sci Med 2004;58:1483-99.

19. Gollac M, Volkoff S. $2^{e}$ ed. Les conditions de travail. Paris : Repères ; 2007.121.

20. François S, Guiho-Bailly M.P, François A, Bodin J, Bertin C, Garré JB et al. Caractéristiques médicales et socioprofessionnelles d'un échantillon de suicidants. $42^{\text {es }}$ journées du Groupement d'Étude et Prévention du Suicide, 20 au 24 septembre 2010. Versailles. France. Disponible à l'adresse URL:http://www.geps. asso.fr/download.php?fname $=$ Francois $S$-TS et travail-geps2010.pdf

21. Cohidon C, Santin G, Geoffroy-Perez B, Imbernon E. Suicide et activité professionnelle en France. Rev Epidemiol Sante Publique 2010;58:139-50.

22. Suicides et tentatives de suicide en France. Études et résultats. Direction de la Recherche, des Études, de l'Évaluation et des Statistiques. 2006(488). 8. Disponible à l'adresse URL:http://www.sante.gouv.fr/ drees/etude-resultat/er488/er488.pdf

23. Takada M, Suzuki A, Shima S, Inoue K, Kasukawa S, Hojoh M. Association between Lifestyle Factors, Working Environment, Depressive Symptoms and Suicidal Ideation:Large-Scale Study in Japan. Ind Health. 2009;47:649-55.

24. Touranchet A, Derriennic F, Volkoff S. Enquête ESTEV : un instrument d'étude des relations entre âge, santé et travail. Arch Mal Prof 1992;53(2):79-89.

25. Chini $B$. Les facteurs de stress professionnel. Enquête auprès des salariés des services interentreprises. Arch Mal Prof 2003;64(5):297-309.

26. Parent-Thirion A, Fernández Macías E, Hurley J, Vermeylen G. Quatrième enquête européenne sur les conditions de travail 2005. Fondation européenne pour l'amélioration des conditions de vie et de travail. Luxembourg, Office des publications officielles des Communautés européennes, 2007.

27. Dejours C. $3^{e}$ éd. Travail, usure mentale. Paris : Bayard ; 2000. 298.

28. Cohidon C. Samotrace point d'étape. Disponible à l'adresse URL: http://www.smt-dauphine-savoie.fr/ 2008-06-20/SocMedW0608.pdf.

29. Davezies P. Suicides : de mauvaises réponses à une vraie question, Santé \& Travail 2007(60) : 29-31.

30. Dejours C. L'évaluation du travail à l'épreuve du réel critique des fondements de l'évaluation. Paris : INRA ; 2003.82.

31. Dejours C. $4^{\mathrm{e}}$ éd. Le facteur humain du travail. Paris : PUF ; 2007.127.

32. Molinier P. $2^{e}$ éd. Les enjeux psychiques du travail. Paris : Payot et Rivages ; 2006. 335.

33. Indicateurs provisoires de facteurs de risques psychosociaux au travail. Collège d'expertise sur le suivi statistique des risques psychosociaux au travail. DARES DREES. 2009.

34. Guiho-Bailly MP, Guillet D. Existe-t-il des situations d'urgence en psychopathologie du travail ? Travailler. 2003(10):57-75.

\section{BIBLIOGRAPHIE INDICATIVE}

Recensement général de la population, Insee : Institut national de la statistique et des études économiques. Disponible à l'adresse URL: www.insee.fr 